UCRL-ID- - 104090

DE91 000073

\title{
REMAP: a computer code that transfers node information between dissimilar grids
}

\author{
Arthur B. Shapiro
}

\section{Lawrence Livermore National Laboratory}

\section{April 1990}

Lawrence Livermore National Laboratory

Mail Stop L-194

7000 East Ave.

Livermore, CA 94550

phone: $415-422-5066$

fax: $415-422-1370$ 


\section{Table of Contents}

1 Introduction 1

2 Execution and Input 3

2.1 Terminal Execution Line 3

2.2 Input Filc 4

2.2.1 Control Card 4

2.2.2 Node Point Data 5

2.2.3 Element Data 7

3 Example 9

3.1 REMAP Examplc 9

3.2 WARNING - Uscr Beware

4 Theory 17

4.1 Coarse Filter 17

4.2 Fine Filter 18

4.3 Shape Functions 20

4.4 Calculating the Natural Coordinates 22

4.5 Temperaturc Interpolation 23

References 25 


\section{Introduction}

REMAP is a computer cede that transfers the axisymmetric, two dimensicial planar, or three dimensional temperature field from one finite element mesh to another. The meshes may be arbitrary as far as the number of elements and their geometry. REMAP int apolates or extrapolates the node temperatures from the old mesh to the new mesh using linear, bilinear, or trilinear isoparametric finite element shape functions. REMAP is used to transfer the temperature field from a thermal analysis mesh to a more finely discretized structural analysis mesh when performing a thermal stress analysis. REMAP was designed to be used with the finite element heat transfer codes TOPAZ2D $|1|$ and TOPAZ3D [2], and the solid mechanics codes NIKE2D [3] and NIKE3D [4]. The I/O formats in REMAP can be easily modified to accept input fiom other codes (e.g., finite difference) and generate output files for other structural codes. REMAP can be used to transfer any scalar field variable between dissimilar finite element meshes.

Three papers by Thomr son from NASA Lewis and Maffeo form GE on the TRANCITS code |5-7| were found useful in developing REMAP. Their idea of a coarse filter followed by a fine filter to determine which element from the old mesh contains a node point from the ne; mesh was used. The coarse filter determines a subset of elements from the old mesh that may contain the new node point. The fine filter determines the element that contains the new node point. REMAP uses the ray-surface intersection algorithm developed for the FACET $[8 \mid$ code for the fine filter. This algorithm has the added capability to determine which element the node is closest to if the node point lies outside the perimeter (or surface) of the old mesh. Once an element from the old mesh has been identified as containing of closest to the new node point, the natural coordinates for the node point are calculated. The isoparametric finite element shape functions are calculated next. These shape functions are then used to interpolate or extrapolate the temperatures from the nodes comprising the old element to the new node point.

Chapter 2 contains information on preparing in input file and running REMAP. Example problems are presented in Chapter 3. The mathematical theory and numerical implementation are presented in Chapter 4. 


\section{Execution and Input}

\subsection{Terminal Execution Line}

REMAP is kept in the public library MDGLIB on all Cray machines. To obtain a copy of REMAP in your private disk space, type

\section{exe mdglib remap $x$.}

The terminal exccution line for REMAP is

$$
\text { remap } i=\inf g=\operatorname{tpf} 0=\text { nikf } p=p f l
$$

where

- inf $=t e x t$ input file containing control information, new node point coordinates, and new element connectivity.

- $\quad t p f=a b s o l u t e$ input file containing old node point coordinates, temperatures, and old element connectivity. This is a TOPAZ plot fite created from running TOPAZ for the thermal analysis, If you do not need to remap, then this file is the temperature input file to be used with NIKE for a thermal stress analosis.

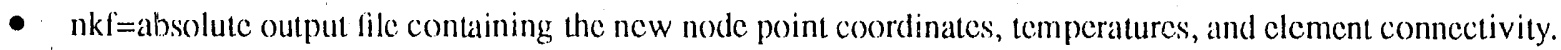
This is the temperature input file to be used with NIKE for a thermal stress analysis on the new mesh. (default - nkplot)

- pfl=text output file listing the input information and temperatures at the node points of the new mesh. (default=printll)

File name dropouis are permitted, for example

\section{remap i=inf $g=t p f$}

The two outpul files, nkf and pfl, will be created with the default names nkplot and printil, respectively. 


\subsection{Input File}

NOTE: Values entered are for the new mesh

\subsubsection{Control Card}

column format

quantity

$1-5 \quad 15 \quad$ number of materials

6-10 $\quad$ is number of node points

$11-15 \quad 15 \quad$ number of elements

16-20 $15 \quad$ geometry type $(1=$ axisymmetric, $2=2 \mathrm{D}$ planar, $3=3 \mathrm{D})$

21-25 $\quad 15 \quad$ temperature flag $(0=$ default; $1=$ use temperatures on the nodal cards as the initial condition) 


\subsubsection{Node Point Data}

The node point data is formatted according to NIKE2D or NIKE3D. Supply one card for each node point with the following information:

NIKE2D - Axisymmetric and 2D-planar Mesh

column format

quantity

$1-5 \quad 15 \quad$ node point number, $\mathrm{n}^{\mathrm{i}}$

6-10 F5.0 displacement boundary condition code, (see NIKE2D users manual [3])

11-20 E10.0 $\quad r^{i}$ coordinate

21-30 E10.0 $\quad z^{i}$ coordinate

31-35 $15 \quad$ node generation increment, $\mathrm{k}$

36-45 E10.0 node paint temperature - if not equal to zero, then this temperature will overwrite the initial temperature in the TOPAZ temperature file

Node point cards do not need to be in order. However, the highest node point number must terrninate the node data. When node data is missing, node numbers are generated according to the sequence

$$
n^{i}, n^{i}+k, n^{i}+2 k, \ldots, n^{j}
$$

where $n^{i}$ and $n^{j}$ are the node numbers defined on two consecutive cards, and $k$ is taken from the first card. Linear interpolation is used to obtain the coordinate of the generated nodes and the node temperatures. The boundary condition code of generated data is set to zero whenever the boundary condition code of $n^{i}$ differs from that of $n^{j}$. 
NIKE3D - 3 Dimensional Mesh

\begin{tabular}{|c|c|c|}
\hline column & format & quantity \\
\hline $1-5$ & 15 & node point number, $n^{i}$ \\
\hline $6-10$ & 15 & $\begin{array}{l}\text { displacement boundary condition code (see NIKE3D users } \\
\text { manual [4]) }\end{array}$ \\
\hline $11-30$ & E20.0 & $x^{\prime}$ coordinate \\
\hline $31-50$ & E20.0 & $y^{\prime}$ coordinate \\
\hline $51-70$ & E20.0 & z. coordinate \\
\hline $71-75$ & 15 & node generation increment, $k$ \\
\hline $76-80$ & 15 & $\begin{array}{l}\text { rotational boundary condition code (see NIKE3D users } \\
\text { manual [4]) }\end{array}$ \\
\hline
\end{tabular}




\subsubsection{Element Data}

The element data is formatted according to NIKE2D or NIKE3D. Supply one card for each element with the following information:

NIKE2D - Axisymmetric and 2D-planar Mesh

column format

quantity

\begin{tabular}{|c|c|c|}
\hline $1-5$ & 15 & element number \\
\hline $6-10$ & 15 & node $n_{i}$ \\
\hline $11-15$ & 15 & node $n_{2}$ \\
\hline $16-20$ & 15 & node $n_{3}$ \\
\hline $21-25$ & 15 & node $n_{4}$ \\
\hline $26-30$ & 15 & material number \\
\hline $31-35$ & 15 & element node point generation increment, $k$ \\
\hline $36-80$ & & $\begin{array}{l}\text { Data in these columns are not read by REMAP. However, this } \\
\text { information may be required for the NIKE } 2 D \text { simulation. }\end{array}$ \\
\hline
\end{tabular}

Element cards are assumed to be in element number sequence. The highest element number must terminate the data. Omitted data is automatically generated with respect to the first card prior to the omitted data by $n^{i+1}=n^{i}+k$. The material property number and element node point generation increment are taken from the first card. The convention for numbering node points is shown below.

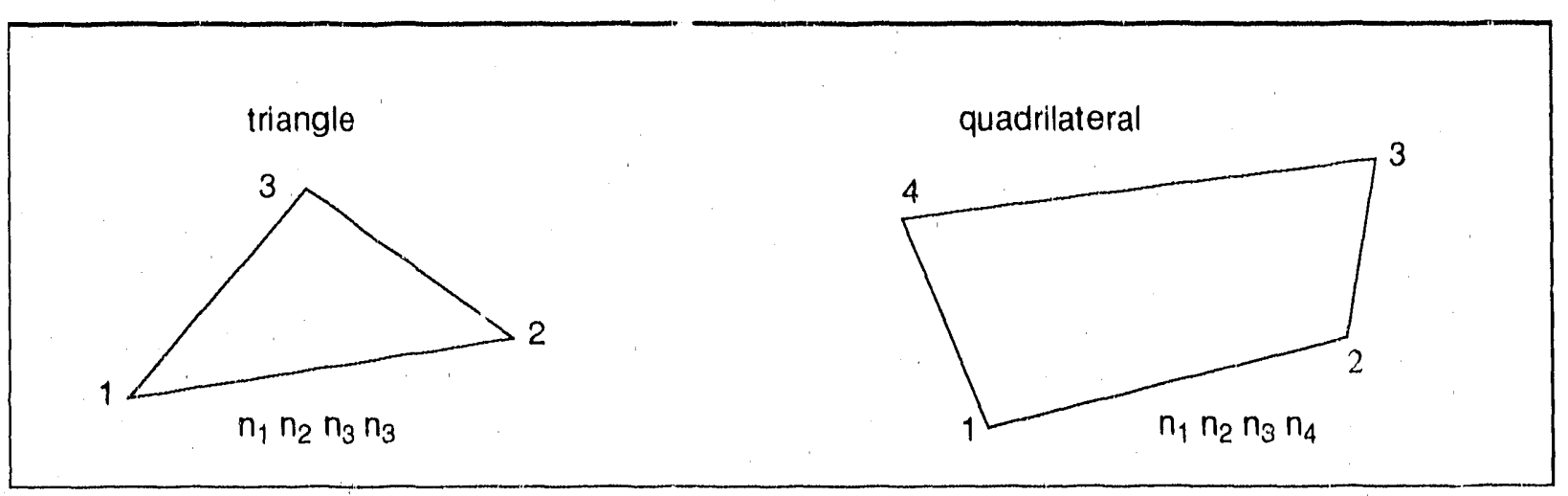


NIKE3D - 3 Dimensional Mesh using 4, 6, or 8 node solid elements

\begin{tabular}{lll} 
column & format & quantity \\
\hline $1-5$ & 15 & element number \\
$6-10$ & 15 & material number \\
$11-15$ & 15 & element node point generation increment \\
$16-20$ & 15 & node $n_{1}$ \\
$21-25$ & 15 & node $n_{2}$ \\
$26-30$ & 15 & node $n_{3}$ \\
$31-35$ & 15 & nod's $n_{4}$ \\
$36-40$ & 15 & node $n_{5}$ \\
$41-45$ & 15 & node $n_{6}$ \\
$46-50$ & 15 & node $n_{7}$ \\
$51-55$ & 15 & node $n_{8}$
\end{tabular}

The convention for numbering 4, 6, and 8 node solid elements are shown below.
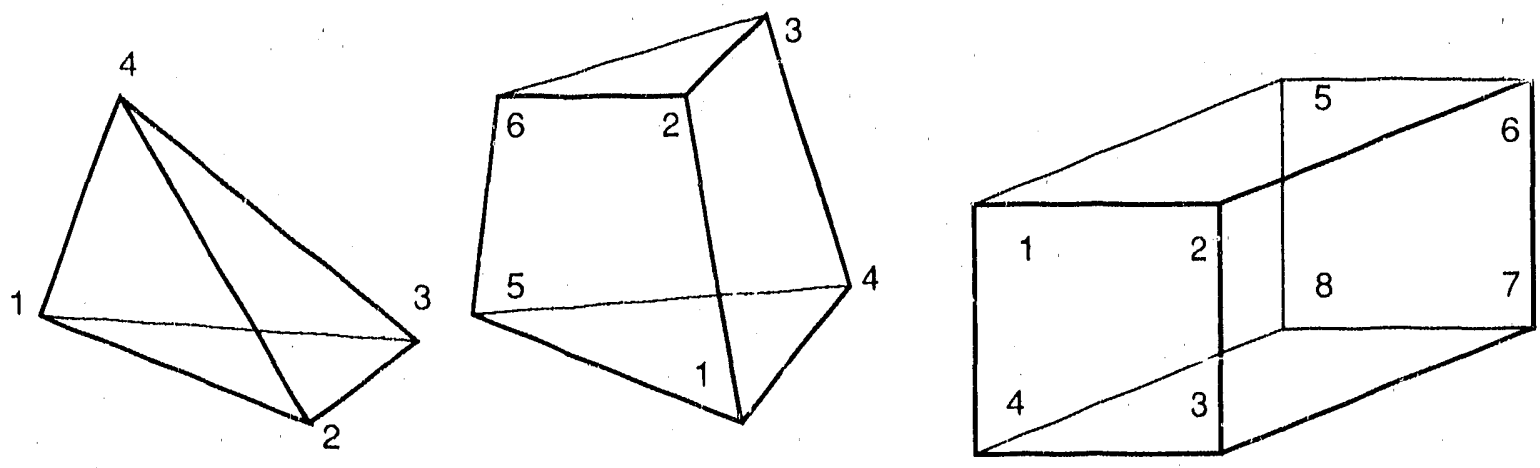

$n_{1} n_{2} n_{3} n_{4} n_{4} n_{4} n_{4} n_{4}$

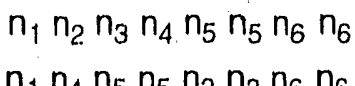

$n_{1} n_{2} n_{3} n_{4} n_{5} n_{6} n_{7} n_{8}$

$n_{1} n_{4} n_{5} n_{5} n_{2} n_{3} n_{6} n_{6}$ 


\section{Example}

\subsection{REMAP Example}

The only intent of the following hypothetical problem is to demonstrate the procedure in using REMAP. There is no physical basis to the problem definition. The objective is to perform a thermal analysis on one mesh and then map the temperature field onto another mesh for the stress analysis. The TOPAZ2D input filc for this problem is shown in Fig 1. The input file defines a 2-dimensional slab, 2 units long by 0.6 units thick, with temperature boundary conditions of $(\mathrm{FF}$ and $2 \mathrm{~F}$ applied to the vertical end surfaces. The lateral surfaces are adiabatic. The material density, heat capacity, and thermal conductivity are all given a value of unity. The finite element mesh is shown in Fig 2 and isothu ins of the calculated node point temperatures are shown in Fig 3. The post processing code ORION $\{10\}$ was used to generate the mesh and isotherm plots using the output plot file, T2PLOT, created by TOPAZ2D.

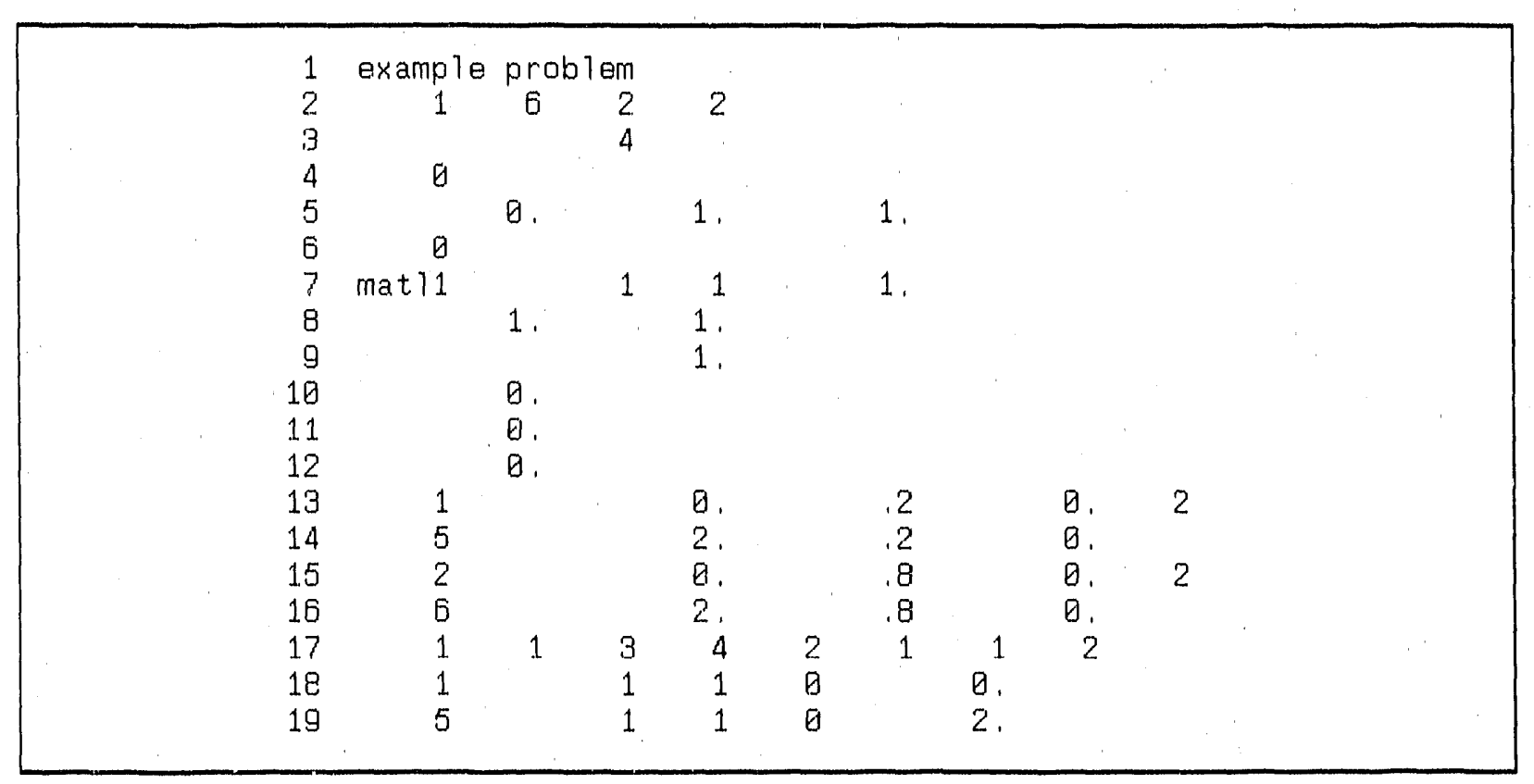

FIGURE 1: TOPAZ2D input file defining the heat transfer problem. 
Example problem

$d s f=0.10$ get 01

$\operatorname{time}=0.100 \mathrm{e}+01$

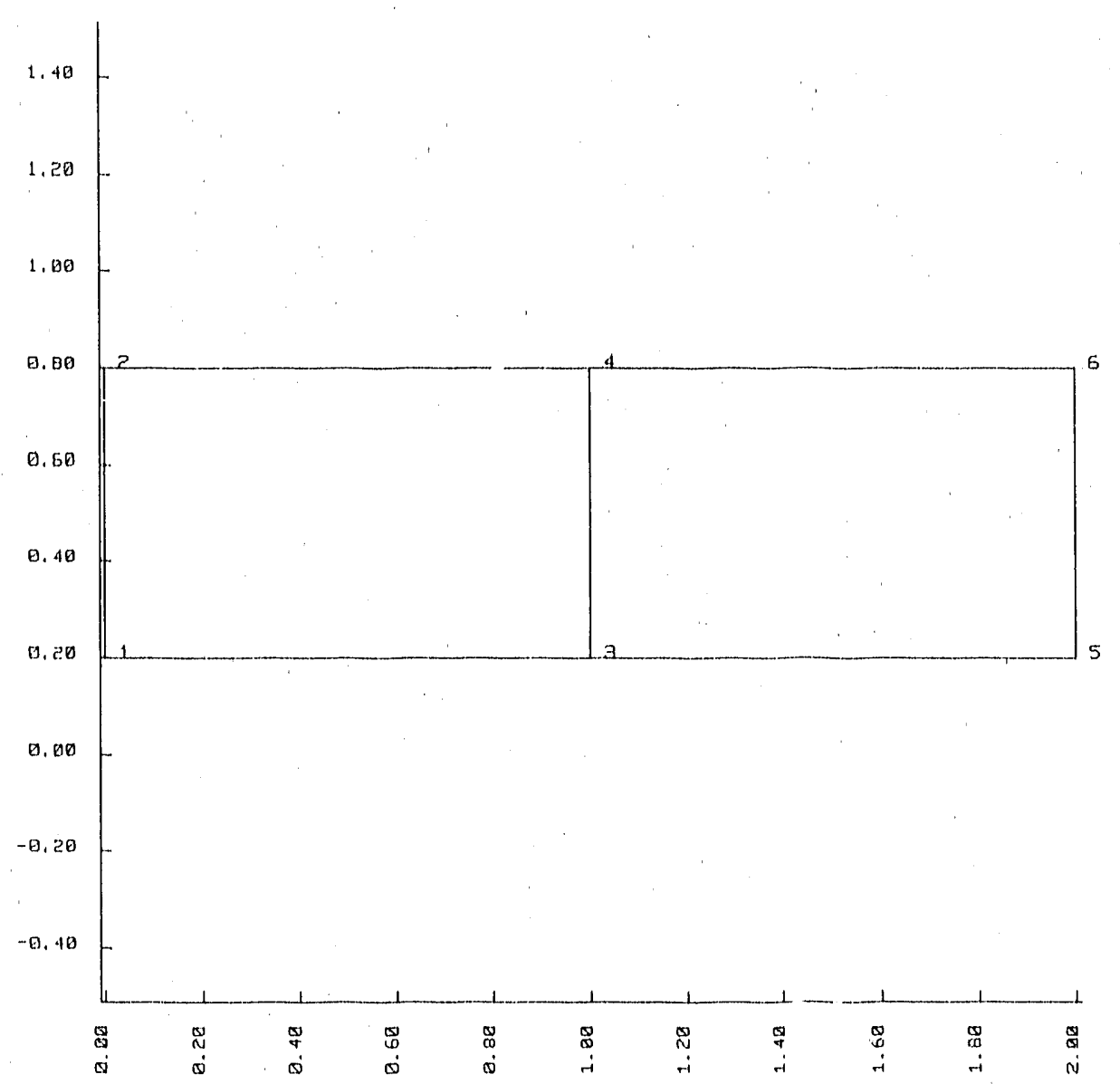

FIGURE 2: The thermial mesh consists of 2 elements and 6 node points. 


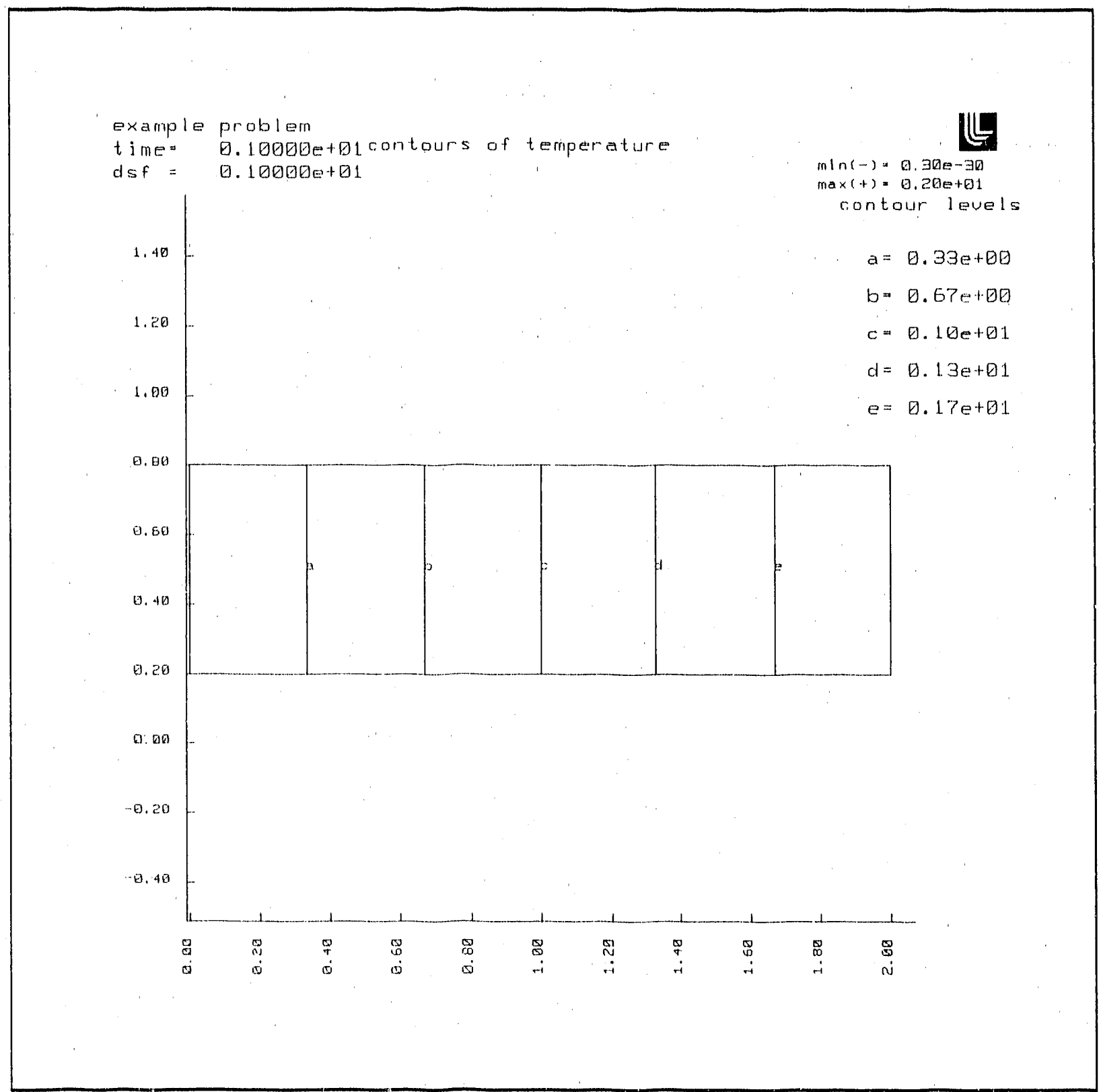

FIGURE 3: Shown is the steady state temperature field. The isotherms are vertical because the heat flow is 1-dimensional from the right surface to the left surface. Also note that the temperature gradient varies linearly between the end surface boundary conditions of OF and $2 F$. 
The REMAP input file, REMAPIN, defining the new mesh for the stress problem is shown in Fig 4 . The execution line for REMAP using the files REMAPIN which defines the stress mesh, and T2PLOT which defines the thermal mesh is

\section{remap $i=$ remapin $g=t 2 p$ lot $/ \mathrm{i} v$}

REMAP creates a fie with the default name NKPLOT. This contains the new mesh data and remapped temperatures to be used as the temperature inpit file to NIKE2D. ORION can be used to display the new mesh (Fig 5) and isotherms of the remapped temperatures (Fig 6). I advise using ORION to plot isotherms on the new mesh and comparing them to isotherms on the old mesh to see if they make sense before performing the thermal stress simulation.

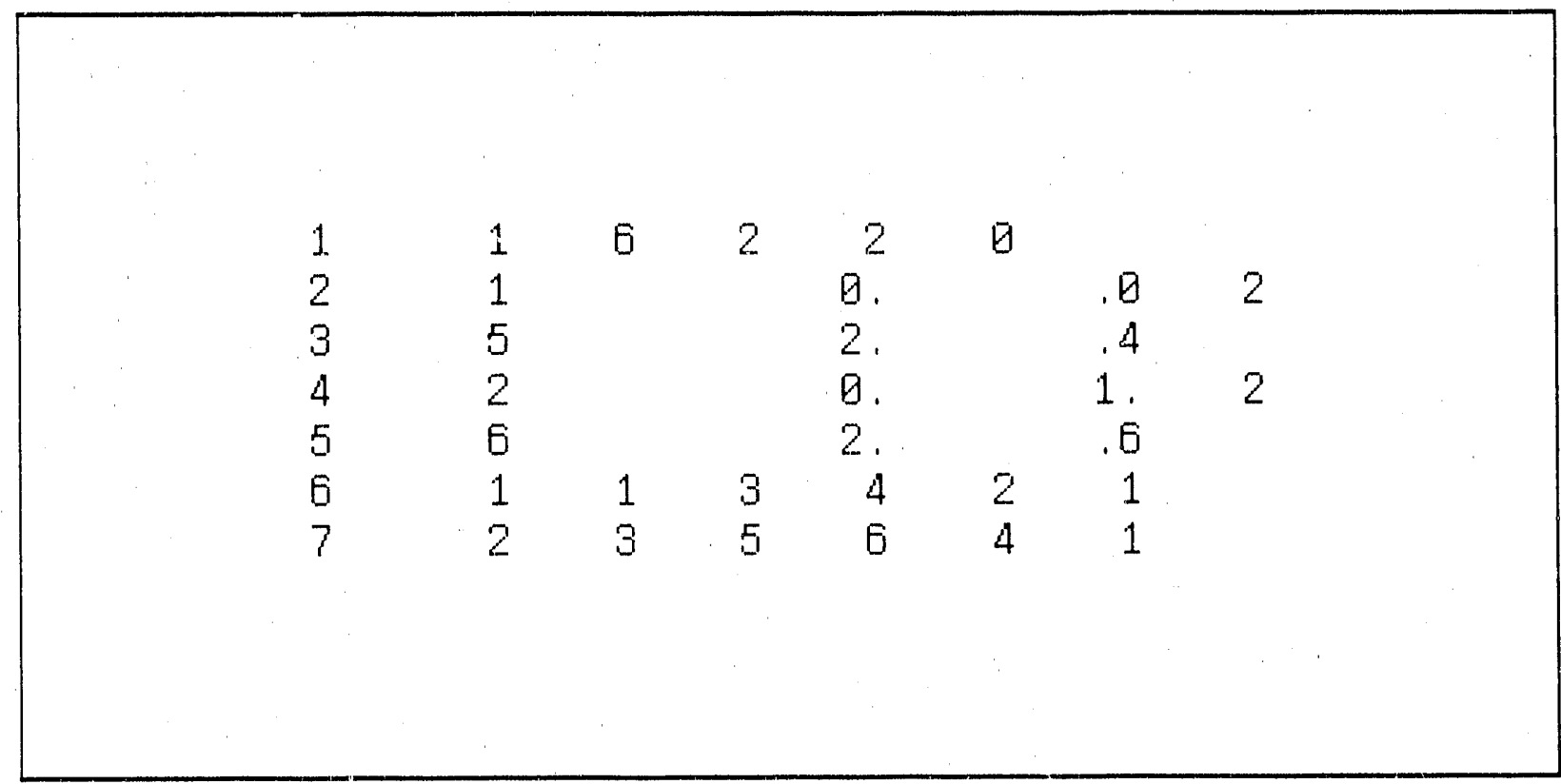

FIGURE 4: The REMAP input file defining the stress mesh. 
example problem

$d s f=0.100 e+01$

time $=0.000$ e+ $\theta$

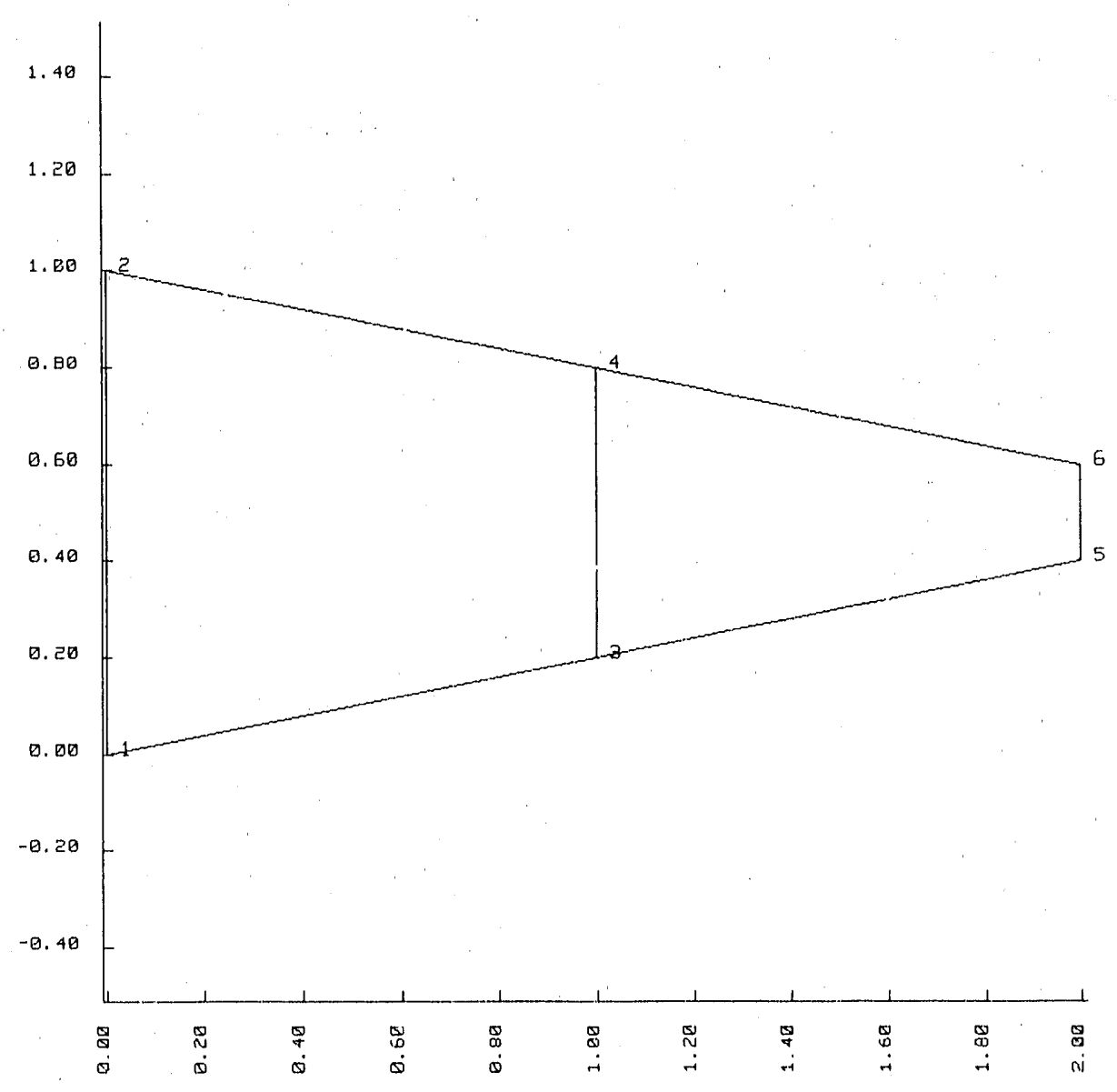

FIGURE 5: The new mesh to be used for the stress problem. The temperatures at node points 1 and 2 will require extrapolation from the thermal mesh (Fig 2), while temperatures at node points 3, 4, 5, and 6 will require interpolation. 
example problem

time $=0.10000 e+01$ contours of temperature

$d s f^{\prime}=$ D. $10000 e+01$

$\ln \ln (-)=0.40 e-30$

$\max (+)=0.20 \mathrm{e}+01$

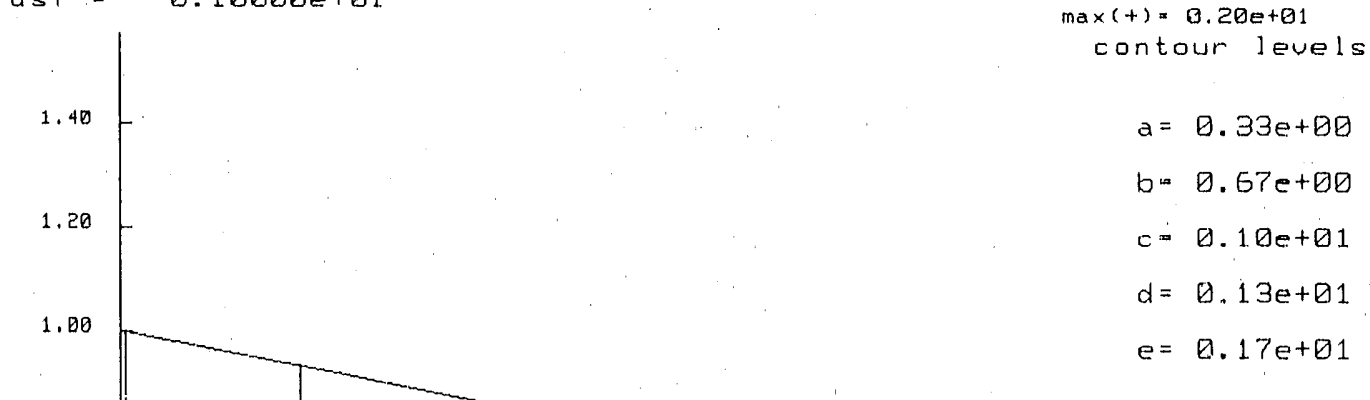

曰. 80

0.68

0.40

0.20

a. 00

$-0.20$

$-0.40$
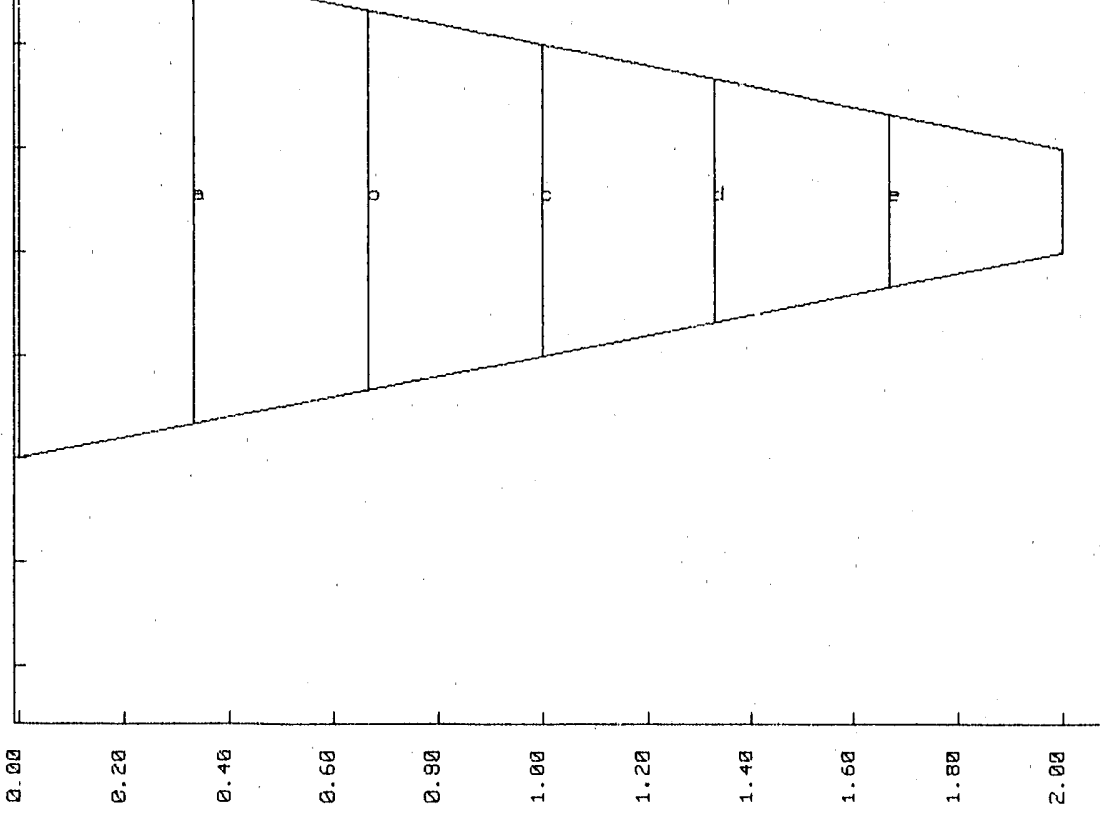

FIGURE 6: Shown is the steady state temperature field mapped onto the stress mesh using the temperature field data from the thermal mesh (Fig 3). 


\subsection{WARNING - User Beware}

The snapping of the temperature field from the thermal mesh (Fig 2) to the stress mesh (Fig 5) is done by interpolation or extrapolation. The governing physics is ignored. Two dimensional heat transfer must be considered to accurately calculate the temperature ficld for the drastically different shane of the stress mesh. Figure 7 shows the isotherms on the stress mesh when 2-dimensional effects are considered. In comparing Fig 7 with Fig 6, note that the isotherms are curved and are shifted to the right. Although this is an extreme example, you should be aware of the physics of the problem that is being remapped.

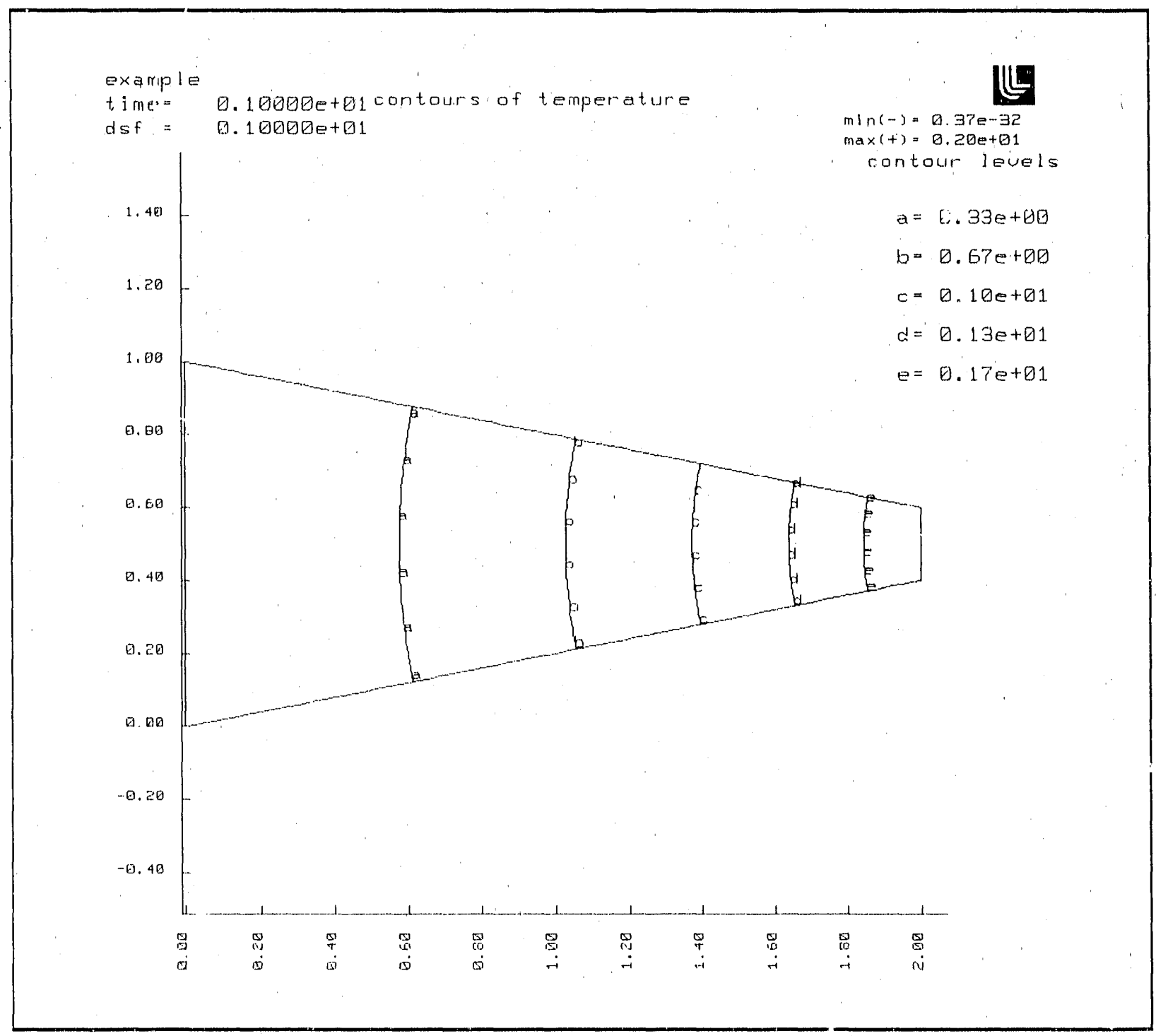

FIGURE 7: Shown are the steady state isotherms on the stress mesh when 2-dimensional heat transfer is considered. Note the difference in shape and position of the isotherms between this figure and Fig 6. 


\section{Theory}

REMAP transfers information (c.g, node point temperature) from an old mesh to a new mesh. To accomplish this, the element from the old mesh containing or closest to a node from the new mesh must be identificd. This is achieved by an element search comprised of a coarse filter followed by a fine filter. The coarse filter uses a simple distance algorithm that identifies a subset of candidate elements. The fine filter determines the one element that contains or is elosest to the new node point. Next, the finite element natural coordinates for the new node point are calculated with reference to the element, followed by the calculation of the isoparametric shape functions. Using these shape functions and the values of the field variable at the element's node points, the value at the new node point is calculated.

Following is a detailed description of the above procedure for two dimensional planar meshes. The two dimensional planar results are directly applicable to an axisymmetric nesh. Only the final equations are given for 3-dimensional meshes. For case of presentation, the following terminology will be followed:

- element refers to the finite clements that comprise the old mesh

- stress point refers to the node points that comprise the new mesh

\subsection{Coarse Filter}

For every stress point (i.c., node point) in the new mesh, the coarse filter searches through all the elements in the old mesh to determine a subset of elements that may contain the stress points. A simple coordinate location check is performed. First, the extreme values of the node point coordinates comprising the element are determined. For a 4 node quadrilateral

$$
\begin{aligned}
& x_{\min }=\min \left(x_{1}, x_{2}, x_{3}, x_{4}\right) \\
& x_{\max }=\max \left(x_{1}, x_{2}, x_{3}, x_{4}\right) \\
& y_{\min }=\min \left(y_{1}, y_{2}, y_{3}, y_{4}\right) \\
& y_{\max }=\max \left(y_{1}, y_{2}, y_{3}, y_{4}\right)
\end{aligned}
$$


Next the stress point coordinates $\left(x_{s}, y_{s}\right)$ are compared to the extreme values of the node points. The stress point is contained by the element if

$$
\begin{aligned}
& x_{\min } \leq x_{s} \leq x_{\max } \\
& y_{\min } \leq y_{s} \leq y_{\max }
\end{aligned}
$$

If the (EQ 2) relationships are satisfied, then the element is included in the subset of possible elements that contain the stress point. As shown in Fig 8, the (EQ 2) conditions are necessary but not sufficient to identify the one element that contains the stress point.

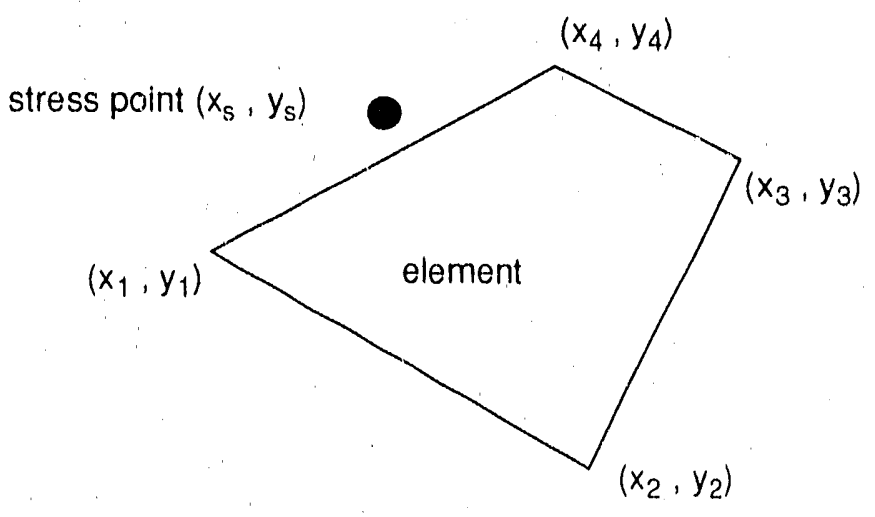

FIGURE 8: A stress point satisfying (EQ 2) but located outside of the element

The z-coordinate must be included in (EQ 1) and (EQ 2) for three dimensional meshes. Additionally, the coordinates of all the element nodes (i.e., 6 for a wedge, 8 for a hexahedral) must be included in determining the extreme values by (EQ 1).

\subsection{Fine Filter}

The fine filter scarches through the subs $\geqslant$ of elements identified by the coarse filter to find the one element that contains the stress point. The symbols used in the following equations are defined in Fig 9.

$$
\begin{aligned}
& \mathbf{V}_{s}=\mathbf{V}_{1}+\alpha_{1} \mathbf{V}_{12}+\alpha_{2} \mathbf{V}_{14} \\
& \mathbf{V}_{s}=\mathbf{V}_{3}+\alpha_{3} \mathbf{V}_{32}+\alpha_{4} \mathbf{V}_{34}
\end{aligned}
$$




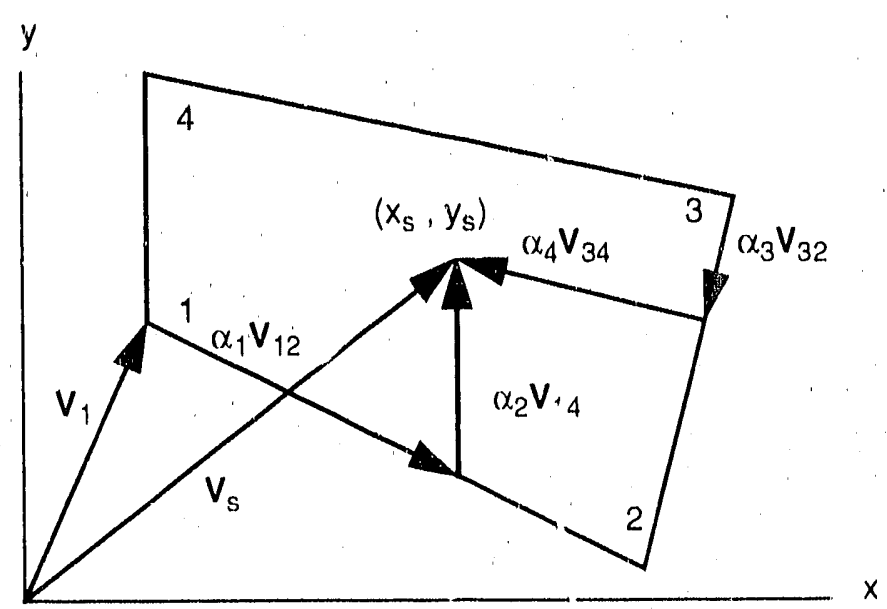

FIGURE 9: his sketch illustrates the symbols used in (EQ 3)

Each of these vector equations can be expanded in terms of their components. 'This provides the following two sets of' simultancous equations to be solved for $\alpha_{1}, \alpha_{2}, \alpha_{3}$, and $\alpha_{4}$.

$$
\begin{aligned}
& {\left[\begin{array}{lll}
x_{2}-x_{1} & x_{4}-x_{1} \\
y_{2}-y_{1} & y_{4} & -y_{1}
\end{array}\right]\left[\begin{array}{l}
\alpha_{1} \\
\alpha_{2}
\end{array}\right]=\left[\begin{array}{l}
x_{w}-x_{1} \\
y_{s}-y_{1}
\end{array}\right]} \\
& {\left[\begin{array}{l}
x_{2}-x_{3} x_{4}-x_{3} \\
y_{2}-y_{3} y_{4}-y_{3}
\end{array}\right]\left[\begin{array}{l}
\alpha_{3} \\
x_{4}
\end{array}\right]=\left[\begin{array}{l}
x_{s}-x_{3} \\
y_{s}-y_{3}
\end{array}\right]}
\end{aligned}
$$

If $\alpha_{1}, \alpha_{2}, \alpha_{3}$, and $\alpha_{4}$ are greater than or equal to $\%$ ero, then the stress point is within or on the surface of the element. A way of mentally visualizing this algorithm is that if the stress point is within both of the angles $\angle 412$ and $\angle 234$, then it is within the element. This algorithm is easily extended into three dimensions. The vector equations for an 8 node hexahedral element (Fig 10) are:

$$
\begin{aligned}
& \mathbf{V}_{s}=\mathbf{V}_{1}+\alpha_{1} \mathbf{V}_{12}+\alpha_{2} \mathbf{V}_{14}+\alpha_{3} \\
& \mathbf{V}_{s}=\mathbf{V}_{3}+\alpha_{4} \mathbf{V}_{37}+\alpha_{5} \mathbf{V}_{34}+\alpha_{6} \mathbf{V}_{32} \\
& \mathbf{V}_{s}=\mathbf{V}_{6}+\alpha_{7} \mathbf{V}_{62}+\alpha_{8} \mathbf{V}_{65}+\alpha_{09} \mathbf{V}_{67} \\
& \mathbf{V}_{s}=\mathbf{V}_{8}+\alpha_{10} \mathbf{V}_{85}+\alpha_{11} \mathbf{V}_{84}+\alpha_{12} \mathbf{V}_{87}
\end{aligned}
$$

Each of these equation can be expanded in terms of their components providing 12 simultancous equations for the 12 unknown $\alpha$ 's. If all $\alpha$ 's are greater than or equat to zero, then the stress point is within or on the surface of the clement. Similar algorithms for a 3 node triangle, a 4 node tetrahedral, and a 6 node wedge are included in REM $\triangle P$. 
If the fine filter does not find an element that contains the stress point, then the stress point lies outside the subsel of elements. The closest element to the stress point is then determined. The sum of the distances between the stress point and the element nodes is calculated. The element for which this sum is a minimum is selected as the element closest to the stress point.

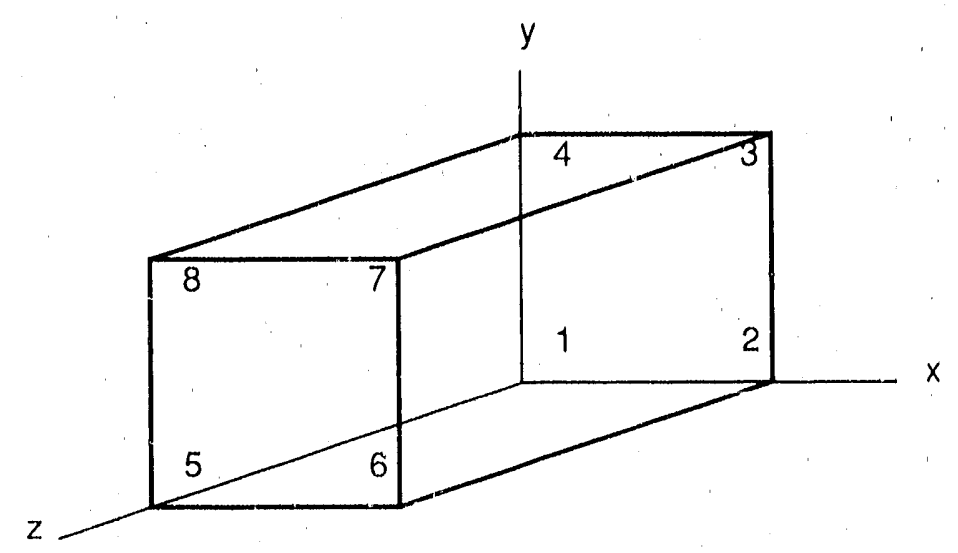

FIGURE 10: Node point numbering for a hexahedral element

\subsection{Shape Functions}

The domain of a straight edged quadrilateral element is defined by the locations of its four node points $\mid\left(x_{a}, y_{a}\right)$, $a=1, \ldots, 4 \mid$. We seck a change of coordinates which maps the given quadrilateral into the biunit square, as depieted in Fig 11. The cerordinates $(\xi, \eta)$ are sometimes called the "natural coordinates". The coordinates $(\xi, \eta)$ of a point in the biunit square are related to the coordinates $(x, y)$ by mappings of the form (see Hughes $|9|$ )

$$
\begin{aligned}
& x(\xi, \eta)=\sum_{a=1}^{4} N_{a}(\xi, \eta) x_{a}^{e} \\
& y(\xi, \eta)=\sum_{a=1}^{4} N_{a}(\xi, \eta) y_{a}^{e}
\end{aligned}
$$

The functions $\mathrm{N}_{\mathrm{a}}$ are the finite element shape functions. For a bilinear quadrilateral they are

$$
\begin{array}{ll}
N_{1}=\frac{1}{4}(1-\xi)(1-\eta) & N_{3}=\frac{1}{4}(1+\xi)(1+\eta) \\
N_{2}=\frac{1}{4}(1+\xi)(1-\eta) & N_{4}=\frac{1}{4}(1-\xi)(1+\eta)
\end{array}
$$



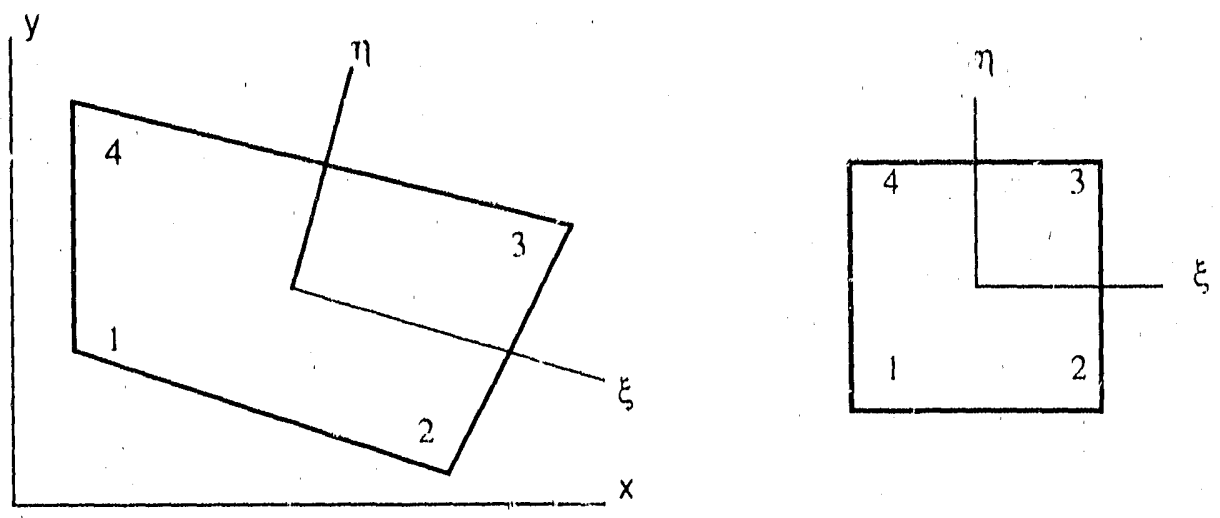

FIGURE 11: Mapping of a quadrilateral into a biunit square

The node numbering for a triangle, 4 node tetrahedral, and 6 node wedge are shown in Fig 12. The nowe numbering for an 8 node hexahedral has previously been shown in Fig 10. The shape functions for a linoar triangle are

$$
N_{1}=\xi \quad N_{2}=\eta \quad N_{3}=1-\xi-\eta
$$

The shape functions for a linear 4 node tetrahedral are

$$
\begin{array}{ll}
N_{1}=\xi & N_{3}=\zeta \\
N_{2}=\eta & N_{4}=1-\xi-\eta-\zeta
\end{array}
$$

The shape functions for a bilincar 6 node wedge are

$$
\begin{array}{ll}
N_{1}=\frac{1}{2}(1-\zeta) \xi & N_{4}=\frac{1}{2}(1+\zeta) \xi \\
N_{2}=\frac{1}{2}(1-\zeta) \eta & N_{5}=\frac{1}{2}(1+\zeta) \eta \\
N_{3}=\frac{1}{2}(1-\zeta)(1-\xi-\eta) & N_{6}=\frac{1}{2}(1+\zeta)(1-\xi-\eta)
\end{array}
$$


The shape functions for a trilinear 8 node hexahedral are

$$
\begin{array}{ll}
N_{1}=\frac{1}{8}(1-\xi)(1-\eta)(1-\zeta) & N_{5}=\frac{1}{8}(1-\xi)(1-\eta)(1+\zeta) \\
N_{2}=\frac{1}{8}(1+\xi)(1-\eta)(1-\zeta) & N_{6}=\frac{1}{8}(1+\xi)(1-\eta)(1+\zeta) \\
N_{3}=\frac{1}{8}(1+\xi)(1+\eta)(1-\zeta) & N_{7}=\frac{1}{8}(1+\xi)(1+\eta)(1+\zeta) \\
N_{4}=\frac{1}{8}(1-\xi)(1+\eta)(1-\zeta) & N_{8}=\frac{1}{8}(1-\xi)(1+\eta)(1+\zeta)
\end{array}
$$
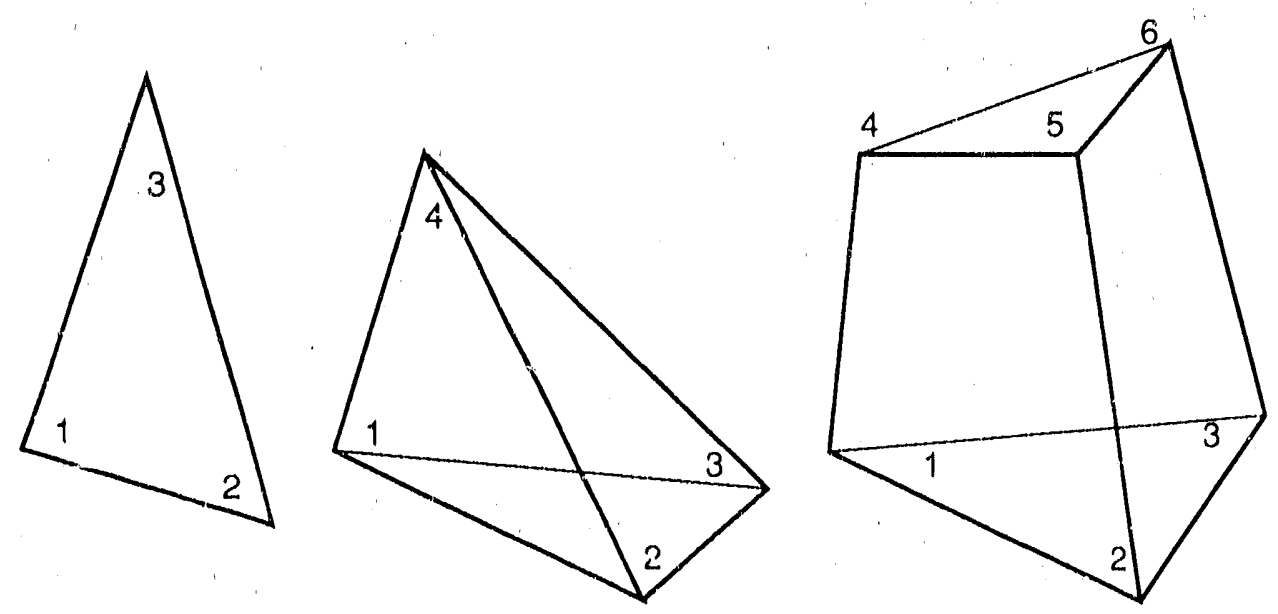

FIGURE 12: Node point numbering for a triangle, tetrahedral, and wedge

\subsection{Calculating the Natural Coordinates}

For a quadrilateral, the natural coordinates corresponding to the stress point can be found by expanding (E丩 6 ) in terms of the shape functions (EQ 7), clement node point coordinates, and stress point coordinates. This provides two nonlinear simultaneous equations that can be solved by Newton's method for $\xi$ and $\eta$.

$$
\begin{aligned}
4 x_{s} & =\left(x_{1}+x_{2}+x_{3}+x_{4}\right)-\left(x_{1}-x_{2}-x_{3}+x_{4}\right) \xi \\
& -\left(x_{1}+x_{2}-x_{3}-x_{4}\right) \eta+\left(x_{1}-x_{2}+x_{3}-x_{4}\right) \xi \eta \\
4 y_{s} & =\left(y_{1}+y_{2}+y_{3}+y_{4}\right)-\left(y_{1}-y_{2}-y_{3}+y_{4}\right) \xi \\
& -\left(y_{1}+y_{2}-y_{3}-y_{4}\right) \eta+\left(y_{1}-y_{2}+y_{3}-y_{4}\right) \xi \eta
\end{aligned}
$$


Note that (EQ 12) can be solved analytically. However, in the computer implementation of the exact solution a decision must be made as to which of the 4 roots should be kept. This decision is casy if the stress point is within the element. Then the roots with values in the range $|-1,1|$ are retained. No clear decision can be made if the stress point is outside the element. Both the exact solution and Newton's method were coded. Newton's method was found to be more reliable in determining the roots of (EQ 12).

The procedure for the linear triangle, linear tetrahedral, 6 node wedge, and 8 noce hexahedral are similar.

\subsection{Tempeiature Interpolation}

The shape functions chosen for the coordinate transformations (EQs 7-11) can be used to interpelate or extrapolate the element node point temperatures to the stress point (i.c., isoparametric concept) by

$$
T_{s}=\sum_{a=1}^{n} N_{a} T_{a}^{e} \quad \text { where } \quad \begin{array}{ll}
n=3 & \text { triangle } \\
n=4 & \text { quadrilateral } \\
n=6 & \text { tetrahedral } \\
n=8
\end{array} \quad \text { hexahedral }
$$




\section{References}

1 A. B. Shapiro, "TOPAZ2D - A Two Dimensional Finite Element Code for Ileal Transfer Analysis, Electrostatics, and Magnetostatic Problems", University of California. Lawrence Livermore National Laboratory, Rept UCID-20824, 1986.

2 A. B. Shapiro, "TOPAZ3D - A Three Dimensional Finite Element Heat Transfer Code", University of California, Lawrence Livermore National Laboratory, Rept UCID-20484, 1985.

3 J. O. Hallquist, "NIKE2D - A Vectorized Implicit, Finite Deformation, Finite Element Code for Analyzing the Static and Dynamic Response of 2-D Solids with Interactive Re\%oning and Graphics", University of California, Lawrence Livermore National Laboratory, Rept UCID-19677, 1986.

4 J. O. Hallquist, "NIKE3D - An Implicit, Finite Deformation, Finite Element Code for Analyzing the Static and Dynamic Response of 3-D Solids", University of California, Lawrence Livermore National Laboratory, Rept UCID $18822,1984$.

5 R. L. Thompson and R. L. Mafleo, "A Computer analysis Program for Interfacing Thermal and Structural Codes", NASA Lewis Research Center, Rept NASA-TM-87(021, 198.5.

6 R. Maffeo, "TRANCITS Program User's Manual", General Electric Company, Cincinnati, OH, NASA Rept CR-174891, 1985.

7 R. Malfeo, "Burner Liner Thermal/Structural Load Modeling", General Electric Company, Cincinnati, OH, NASA Rept CR-174892, 1984.

8 A. B. Shapiro, "FACET - A Radiation View Factor Computer Code for Axisymmetric, 2D Planar, and 3D Geometries with Shadowing", University of Califormilı, Lawrence Livermore National Laboratory, Rept UCID-19887, 1983.

9 T. J. R. Hughes, The Finite Element Method, Prentice Hall, 1987.

10 J. O. Hallquist, "ORION - An Interactive Color Post processor for 2-Dimensional Finite Element Codes", University of California, Lawrence Livermore National Laboratory, Rept UCID-19310, 1985. 

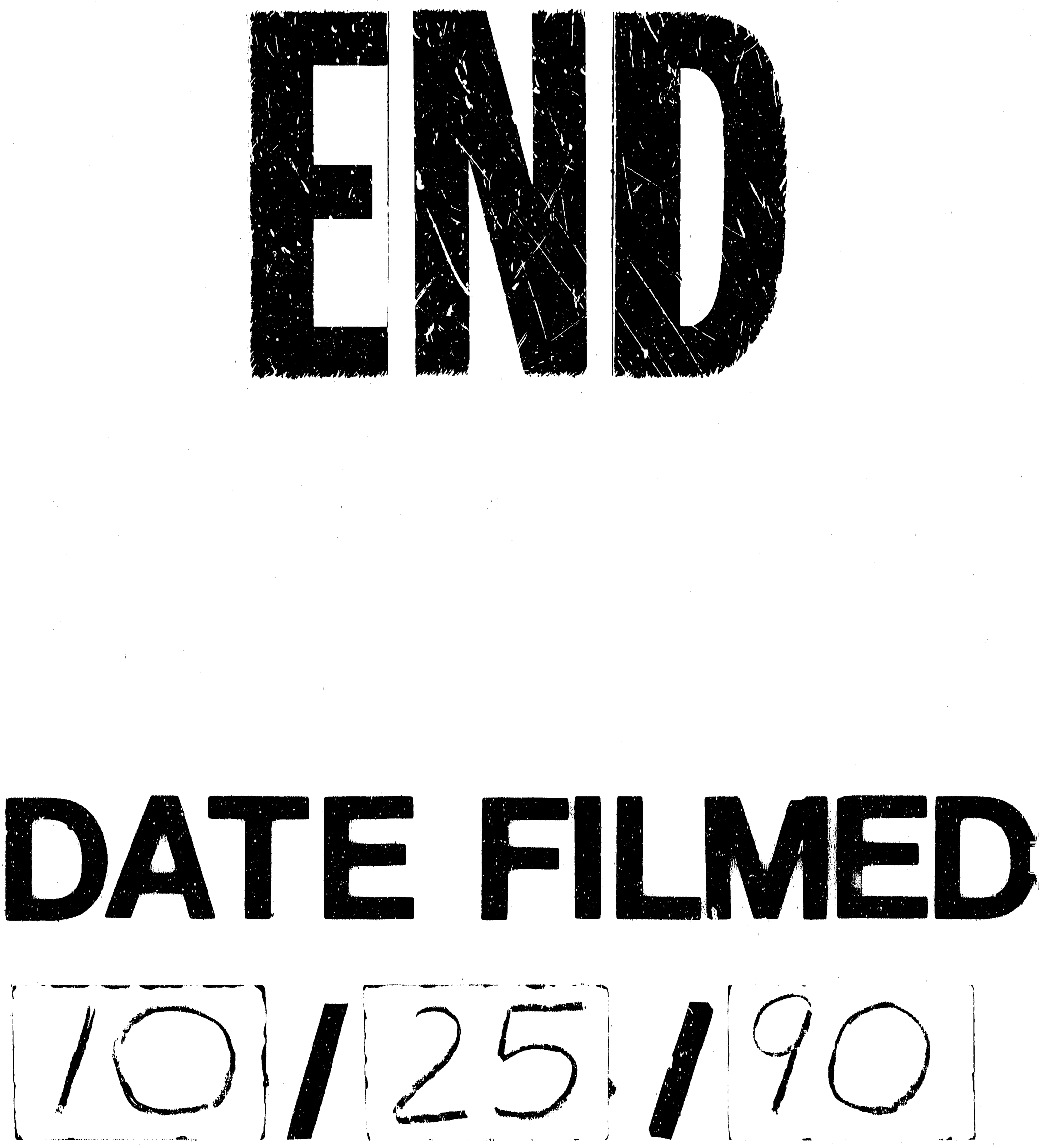

$-$ 
